\title{
Unsteady Natural Convection between Two Eccentric Hemispheres
}

\author{
Mariama Néné Koita1,2, Mamadou Lamine Sow ${ }^{1,2}$, Omar Ngor Thiam², \\ Vieux Boukhaly Traoré1, Cheikh Mbow ${ }^{1,2}$, Joseph Sarr ${ }^{1,2}$
}

\author{
${ }^{1}$ Groupe de Recherche sur l'Energie Solaire et les Transferts (GREST), Faculté des Sciences et Techniques, Université Cheikh Anta \\ DIOP (UCAD), Dakar, Sénégal \\ ${ }^{2}$ Laboratoire de Mécanique des Fluides, Faculté des Sciences et Techniques, Université Cheikh Anta DIOP (UCAD), Dakar, \\ Sénégal \\ Email: mariamanene.koita@ucad.edu.sn
}

How to cite this paper: Koita, M.N., Sow, M.L., Thiam, O.N., Traoré, V.B., Mbow, C. and Sarr, J. (2021) Unsteady Natural Convection between Two Eccentric Hemispheres. Open Journal of Applied Sciences, 11, 177-189.

https://doi.org/10.4236/ojapps.2021.112012

Received: January 1, 2021

Accepted: February 20, 2021

Published: February 23, 2021

Copyright (c) 2021 by author(s) and Scientific Research Publishing Inc. This work is licensed under the Creative Commons Attribution International License (CC BY 4.0).

http://creativecommons.org/licenses/by/4.0/

\begin{abstract}
Natural convection heat transfer in open or closed cavities takes place in different engineering areas. The hemispherical cavity is a part of basic geometries although it is not widely studied. The present paper reports the numerical study of natural convection in a closed hemispherical annulus delimited by two vertically eccentric hemispheres filled with Newtonian fluid (air in this case with $P r=0.7$ ) is conducted. The inner hemisphere is heated by a heat flux of constant density and the outer one is maintained isothermal. Based on the Boussinesq assumptions, the governing equations are numerically studied using unsteady natural convection formulated with vorticity and stream-function variables. These equations are written by using bispherical coordinates system and solved by using a finite difference method. The effect of the control parameters such as the Rayleigh number $\left(10^{3} \leq R a \leq 10^{6}\right)$ or the eccentricity ( $e$ $= \pm 0.2, \pm 0.5,0)$ in the dynamic and thermal behaviours of the fluid is investigated.
\end{abstract}

\section{Keywords}

Natural Convection, Hemispherical Cavity, Rayleigh Correlations, Eccentricity, Nusselt Number

\section{Introduction}

For several decades, heat transfer by natural convection has been the subject of much research and also offers a diverse field of application as electronics, nuclear industry, building or solar energy. Many theorical and experimental studies 
on rectangular [1] [2] or square cavities [3] have been treated given their simplicity of implementation. Other studies have addressed concentric cylinders and spheres. Thus, by studying transient natural convection heat transfer between concentric and vertically eccentric spheres, Chiu and Chen [4] observed that for a range of Rayleigh numbers $\left(10^{3}<R a<10^{5}\right)$, for a Prandtl number and a radius ratio equal respectively 0.7 and 2 , the heat and flow fields are dependent on the Rayleigh number and the eccentricity of the annulus. Crawford and Lemlich [5] analysed natural convection in horizontal concentric cylindrical annuli, they showed that the results revealed characteristic twin kidney-shaped circulation patterns. The two-dimensional heat transfer induced by free laminar convection in an enclosure is numerically investigated by Sarr, et al. [6]. Their results showed that maximum heat is found when the Grashof number is up to $10^{3}$. The average Nusselt increases with the Prandtl number. Numerical studies have been done in other geometry cavities. Mack and Hardee [7] studied natural convection between concentric at low Rayleigh numbers. They discussed and illustrated streamline configuration, velocity and temperature distributions, and both local and overall heat transfer rates. They showed that negative eccentricities have been to enhance convection while positive eccentricities have the reverse effect and the heat transfer actually increases slightly for very high positive eccentricities where conduction plays an important role. For their part, Sow et al. [8] by studying geometrical and Rayleigh number effect free convection between two vertically eccentric spheres confirms that the convection motion is reinforced for the geometries characterized by positive values of the eccentricity with heat exchange increasing and the fluids flow depends strongly on the eccentricity and the modified Rayleigh number. In these different works which the geometry change, the authors conducted these studies on the influence of Rayleigh or Grashof number, the eccentricity and the ratio of radii. However, very little studies are done on natural convection in hemisphere. Some of documents with this problem are Shiina, et al. who studied natural convection heat transfer in hemisphere with bottom surface heated and hemispherical surface cooled, the heat transfer is quantified by means of Nusselt-Rayleigh-type correlations. Lewandowski, et al. [9] examined natural convection from isothermal hemisphere, they found a correlation between the Nusselt and Rayleigh number which is a quarter. Bairi, et al. [10] dealt with isothermal disk and dome faced downwards. Their results show that the natural convective heat transfer are between $13 \%$ and $21 \%$ higher when the disk is maintained isothermal as compared to the case corresponding to imposed heat flux on the disk, for the same inclination angle and Rayleigh range. The numerical study was treated by Alilat [11] with inclined concentric hemispheres quantifies the convective heat transfer. His results show that the inclination angle of device affects only weakly the heat exchange. According to the authors' knowledge, research concerning natural convection between two eccentric hemispheres is relatively limited. The purpose of the present paper is to report an investigation of transient natural convection between two 
hemispheres inclined with the inner hemisphere heated by a heat flux of constant density and the outer one is maintained isothermal. The governing equations are formulated in terms of vorticity and stream function.

\section{Materials and Methods}

\subsection{Problem Definition}

Consider as depicted in Figure 1 a motion of a Newtonian fluid (air in this case) confined within an annular space enclosed delimited by two vertically eccentric hemispheres whose radii are $R_{i}$ and $R_{e}$ centered respectively on $\theta_{i}$ and $\theta_{e}$. The eccentricity $e^{\prime}$ is defined as the algebraic distance separating the two centers of hemispheres.

Initially, the enclosure is at a uniform temperature. From this time, a constant wall heat flux $(q)$ is suddenly applied on the inner hemisphere while the outer is maintained isothermal $\left(T_{c t e}^{\prime}\right)$. The walls separating the two hemispheres $(\theta=0$, $\theta=\pi)$ are insulated. The two hemispheres being heated differently, a transient convection developpes inside the enclosed space.

We assume that the physical properties of the fluid are constant unless this density in terms of gravity, which in a first approximation of Boussinesq hypothesis, varies linearly with temperature. Viscous dissipation, thermal radiation and compressibility effects are neglected. We considered the fluid Newtonian and the flow incompressible and bidimensionnal. In order to reduce the curvilinear enclosure into a rectangular field a curvilinear coordinate system is used. A conformal transformation [12] makes it easier to write expressions of boundary conditions. It's a system which is constituted by families of eccentric spheres whose centres are located on vertical axis $(o y)$ and by poles $(+a)$ and $(-a)$. The passage of Cartesian coordinate $(x, y)$ to the bispherical coordinate $(\eta, \theta)$ is carried out using the following by the formulas:

$$
\left\{\begin{array}{l}
x=\frac{a \sin \theta}{\operatorname{ch} \eta-\cos \theta} \\
y=\frac{a \operatorname{sh} \eta}{\operatorname{ch} \eta-\cos \theta}
\end{array}\right.
$$

The walls located on the vertical axis are given by $\theta=0$ and $\theta=\pi$. The inner

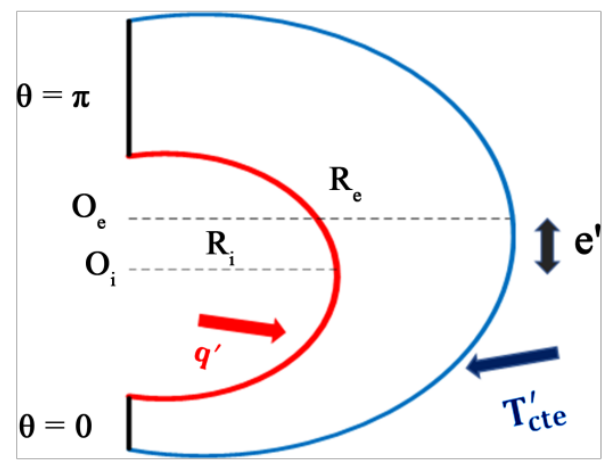

Figure 1. Geometry of the problem. 
hemisphere is materialized by line of coordinate $\left(h=h_{i}\right)$ and the outer hemisphere $\left(h=h_{e}\right)$.

Under these assumptions, the equations governing the problem in a dimensionless vorticity-stream function are the equation of the momentum and heats equations respectively:

$$
\begin{gathered}
\quad \frac{\partial}{\partial t}\left(\frac{\Omega}{K}\right)+\frac{1}{H}\left[U-\frac{3 \operatorname{Prg}_{2}}{K}\right] \partial_{\eta}\left(\frac{\Omega}{K}\right)+\frac{1}{H}\left[V+\frac{3 P r g_{1}}{K}\right] \partial_{\theta}\left(\frac{\Omega}{K}\right) \\
=\frac{P r}{H^{2}}\left[\partial_{\eta}^{2}\left(\frac{\Omega}{K}\right)+\partial_{\theta}^{2}\left(\frac{\Omega}{K}\right)\right]+\frac{R a P r}{K H}\left(G_{2} \partial_{\eta} T-G_{1} \partial_{\theta} T\right) \\
\partial_{t} T+\frac{1}{H}\left[U-\frac{g_{2}}{K}\right] \partial_{\eta} T+\frac{1}{H}\left[V+\frac{g_{1}}{K}\right] \partial_{\theta} T=\frac{1}{H^{2}}\left[\partial_{\eta}^{2} T+\partial_{\theta}^{2} T\right]
\end{gathered}
$$

With:

$$
\begin{aligned}
& \left\{\begin{array}{l}
x=\frac{a \sin \theta}{\operatorname{ch} \eta-\cos \theta} \\
y=\frac{a \operatorname{sh} \eta}{\operatorname{ch} \eta-\cos \theta}
\end{array}\right. \\
& \left\{\begin{array}{l}
U=\frac{1}{K H} \frac{\partial \Psi}{\partial \theta} \\
V=-\frac{1}{K H} \frac{\partial \Psi}{\partial \eta}
\end{array}\right. \\
& \left\{\begin{array}{l}
g_{1}=\frac{1-\cos \theta}{\operatorname{ch} \eta-\cos \theta} \\
y=-\frac{\sin \theta \operatorname{sh} \eta}{\operatorname{ch} \eta-\cos \theta}
\end{array}\right.
\end{aligned}
$$

We define the stream-function which intrinsically verifies incompressibility condition and which has the dimension of a volume flow.

$$
\Psi=K \Phi
$$

is the stream function which has a dimension of a surface flow.

The equation of stream function is given as:

$$
\Omega=\frac{1}{K^{2} H}\left[g_{2} \partial_{\eta} \Phi-g_{1} \partial_{\theta} \Phi\right]-\frac{1}{K H^{2}}\left[\partial_{\eta}^{2} \Phi-\partial_{\theta}^{2} \Phi\right]
$$

The above equations are complemented by the following initial and the boundary conditions:

For $t=0$

$$
\Omega=\Psi=T=U=V=0
$$

And for $t>0$ :

- On the inner hemisphere: $\eta=\eta_{i}$

$$
U=V=\Psi=0
$$




$$
\begin{gathered}
\Omega=-\frac{1}{K H} \partial_{\eta}^{2} \Psi \\
\partial_{\eta} T=H_{i}=\frac{\operatorname{ch} \eta_{i}}{\operatorname{sh}^{2} \eta_{i}}
\end{gathered}
$$

- On the outer hemisphere: $\eta=\eta_{e}$

$$
\begin{array}{r}
U=V=\Psi=0 \\
\Omega=-\frac{1}{K H} \partial_{\eta}^{2} \Psi
\end{array}
$$

- On the vertical walls $\theta=0$ and $\theta=\pi$ :

$$
\begin{gathered}
U=V=\Psi=0 \\
\partial_{\eta} T=0 \\
\Omega=-\frac{1}{K H} \partial_{\theta}^{2} \Psi
\end{gathered}
$$

The heat energy transmitted by the active walls is characterized by the Nusselt number. The local and the average Nusselt numbers relative to the inner and the outer hemisphere are as follows:

- For the inner hemisphere:

$$
\begin{gathered}
N u_{i}=\frac{1}{T_{i, m}} \\
\overline{N u_{i}}=\frac{1}{S_{i}} \int N u_{i} \mathrm{~d} S_{i}
\end{gathered}
$$

- For the outer hemisphere:

$$
\begin{aligned}
& N u_{i}=\frac{1}{H_{e} T_{i, m}} \partial_{\eta} T \\
& \overline{N u_{e}}=\frac{1}{S_{e}} \int N u_{e} \mathrm{~d} S_{e}
\end{aligned}
$$

\subsection{Numerical Procedure}

The implicit method alternating direction (A.D.I) [13] is used for time integration with momentum and heat equations and the finite difference scheme is chosen for spatial integration. The system of linear equations obtained by using A.D.I is solved by Thomas algorithm. The equation of stream function is solved by using the Successive Over Relaxation (S.O.R) [14] with an optimum relaxation parameter.

At each instant, we assume that variable converges to its numerical value if the following stop criterion is satisfied:

$$
\frac{\left|W^{n+1}-W^{n}\right|_{\max }}{\left|W^{n+1}\right|_{\max }} \leq 10^{-5}
$$

where $n$ denotes the iteration step and $W$ stands for $\Omega, \Psi$ and $T$. 


\section{Results and Discussion}

\subsection{Computation Conditions}

Tests have been done on the influence of the mesh and on the time step. Table 1 and Table 2 define the computation conditions respectively compared to the choice of the time step and the mesh. We retain $51 \times 51$ for the grid system and $10^{-5}$ for the time which constitutes a good compromise between a precision and an acceptable computation time.

\subsection{Validation}

Table 3 shows the average Nusselt number computed for various Rayleigh numbers in comparison with those authors. Our results are compared with those published by Tazi, et al. [15] with an axisymmetric steady laminar thermal natural convection in a spherical annulus and Sow, et al. [8] a transient laminar convection between two vertically eccentric spheres. We discovered excellent agreement with them. Therefore, the relative difference between calculated mean Nusselt numbers

Table 1. Effects of time steps on the Nusselt number of the heat wall for $R a=10^{5}$, $e=0, \Delta t=10^{-5}$ and the grid system is $51 \times 51$.

\begin{tabular}{cccc}
\hline & \multicolumn{3}{c}{ Time steps } \\
\cline { 2 - 4 } & $10^{-4}$ & $10^{-5}$ & $10^{-6}$ \\
\hline$N u$ & 4.7751 & 4.9524 & 5.0033 \\
Difference (\%) & 4.56 & 1.02 & 0 \\
Time computing (min) & 24 & 95 & 471 \\
\hline
\end{tabular}

Table 2. Effects of mesh refinement on the Nusselt number of the heat wall for $R a=10^{5}$, $e=0$ and $\Delta t=10^{-5}$.

\begin{tabular}{ccccccccc}
\hline & \multicolumn{8}{c}{ Mesh grid } \\
\cline { 2 - 9 } & $21 * 21$ & $21 * 41$ & $41 * 41$ & $41 * 51$ & $41 * 81$ & $51 * 51$ & $51 * 81$ & $81 * 81$ \\
\hline$N u$ & 5.0217 & 5.0617 & 4.9614 & 4.9647 & 4.9524 & 4.9670 & 4.9624 & 4.9618 \\
Difference (\%) & 1.82 & 2.63 & 0.60 & 0.67 & 0.71 & 0.42 & 0.38 & 0 \\
$\begin{array}{c}\text { Time computing } \\
\text { (min) }\end{array}$ & 7 & 12 & 37 & 45 & 70 & 75 & 145 & 232 \\
\hline
\end{tabular}

Table 3. Comparaison of the mean Nusselt number in a case of $e=0$.

\begin{tabular}{cccccc}
\hline & \multicolumn{5}{c}{$\boldsymbol{R} \mathbf{a}$} \\
\cline { 2 - 6 } & $\mathbf{1 0 ^ { 3 }}$ & $\mathbf{1 0 ^ { 4 }}$ & $\mathbf{1 0 ^ { 5 }}$ & $10^{6}$ & $10^{7}$ \\
\hline$N u$ (our results) & 2.125 & 3.0651 & 4.982 & 7.6874 & 11.671 \\
$N u$ (Tazi et al., 1997) & 2.098 & 3.15 & 5.034 & 7.794 & 12.109 \\
Difference (\%) & 1.76 & 2.70 & 1.03 & 1.37 & 3.62 \\
$N u$ (Sow et al., 2007) & 2.062 & 3.062 & 4.977 & 7.7720 & 12.109 \\
Difference (\%) & 3.54 & 0.10 & 0.10 & 0.42 & 3.62 \\
\hline
\end{tabular}


was less than $3.7 \%$ for all cases presented.

\subsection{Evolution of Isotherms and Streamlines}

Figures 2(a)-(c) present side by side for each value of eccentricity the time evolution of the isotherms and the streamlines. The eccentricity varies from -0.5 to +0.5 and the Rayleigh number and the Prandtl number are equal respectively to $10^{6}$ and 0.7 .

The shape of isothermal lines initially matches the internal wall during the first moment. However the isotherms are increasingly deformed over time for all the studies values of eccentricities. In general, the fluid heats up as it rises along

(a)
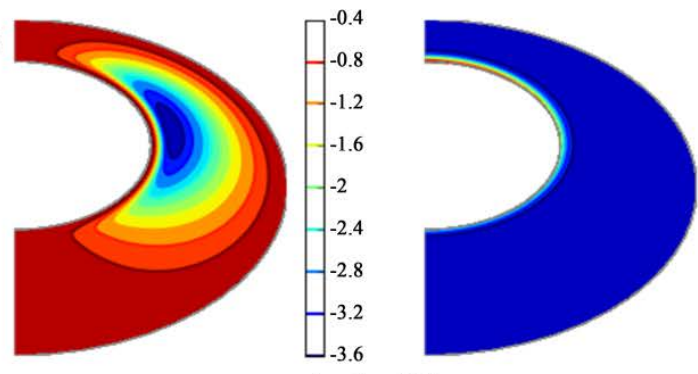

$\mathrm{t}=5 \times 10^{-3}$
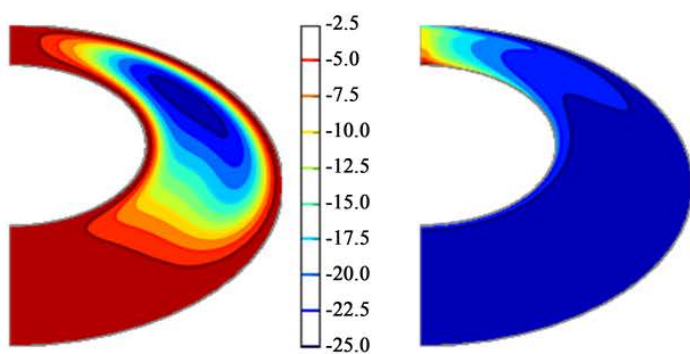

$\mathrm{t}=7 \times 10^{-1}$

(b)
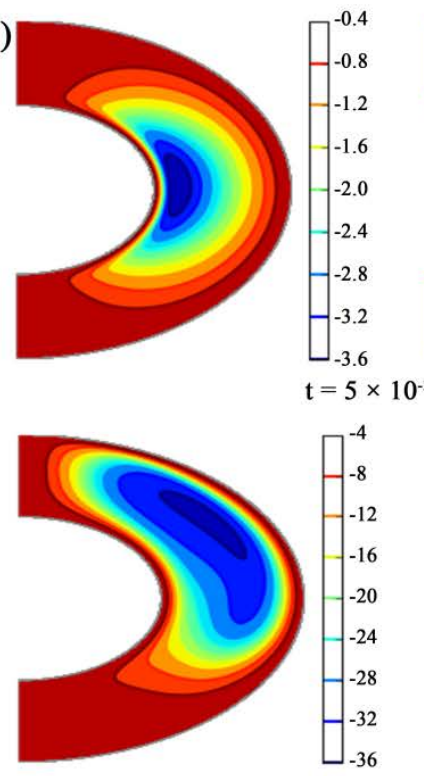

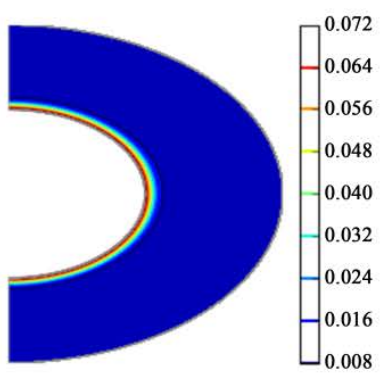

-8
-8
-12
-16
-20
-24
-28
-32
-36 $\mathrm{t}=7 \times 10^{-1}$
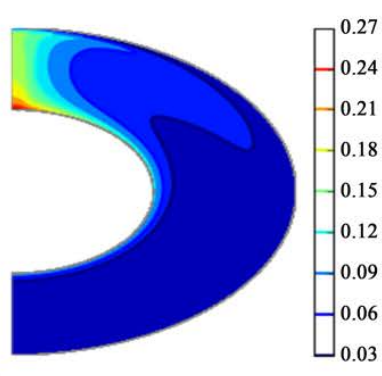
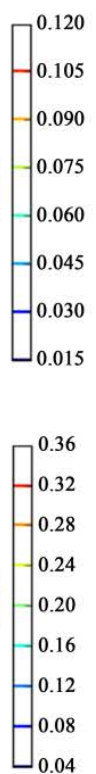
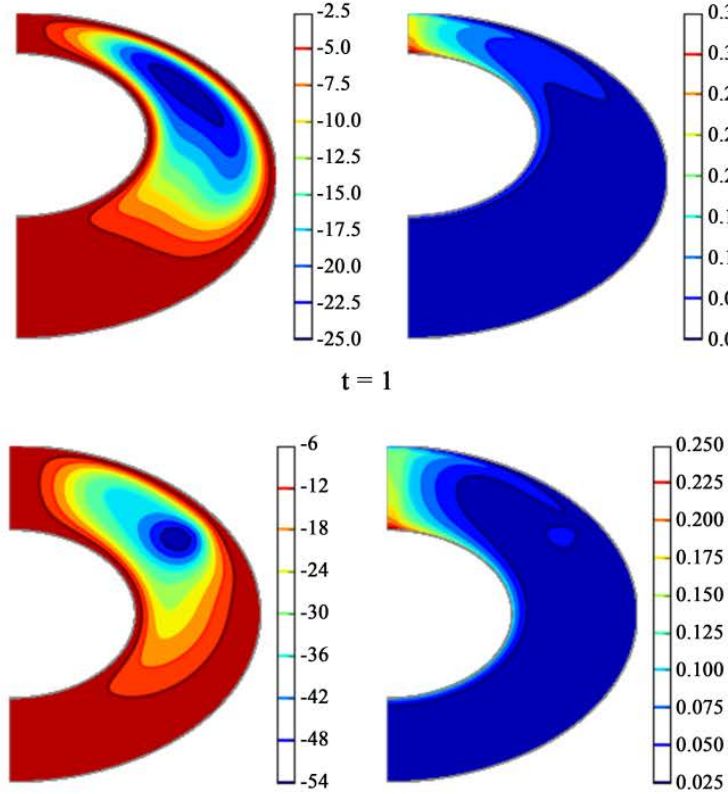

$\mathrm{t}=4 \times 10^{-2}$
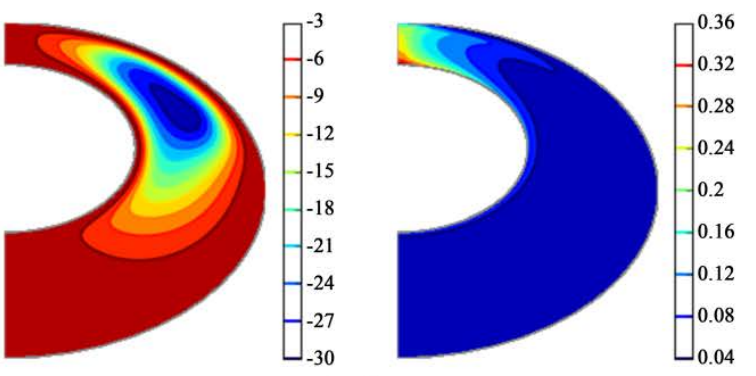

$\mathrm{t}=1$
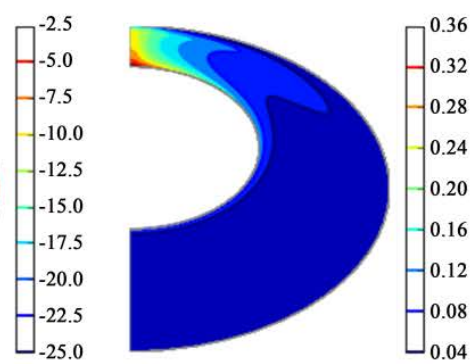

$\mathrm{t}=1$

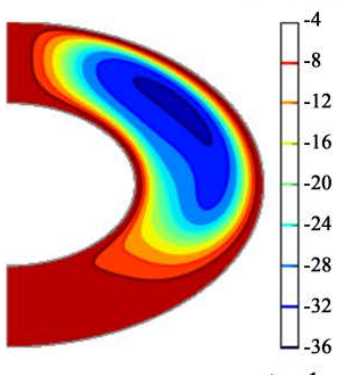

$\mathrm{t}=1$

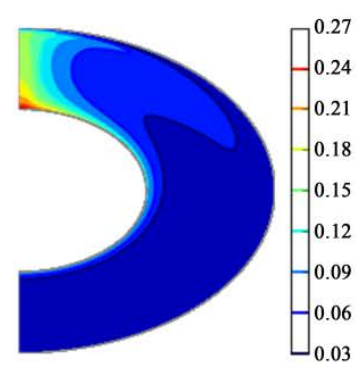


(c)

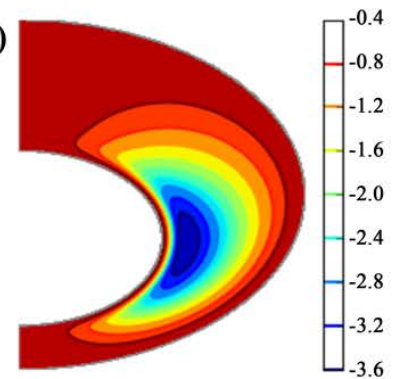

$\mathrm{t}=5 \times 10^{-3}$

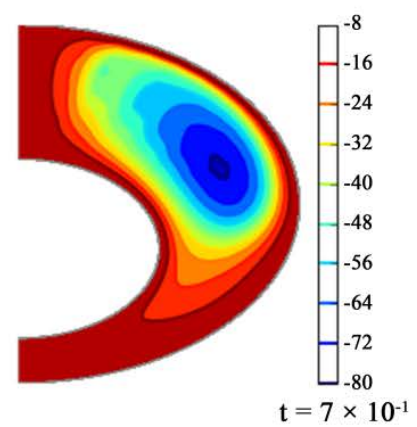

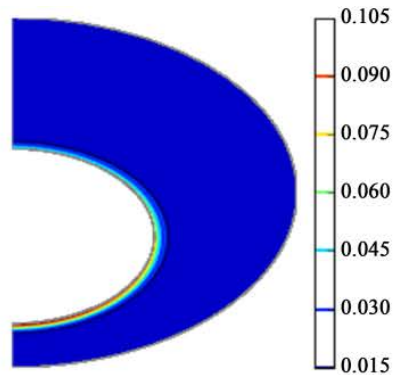

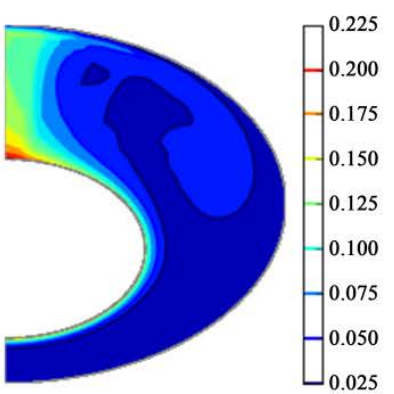

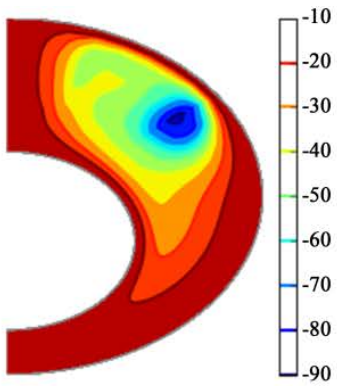

$\mathrm{t}=4 \times 10^{-2}$

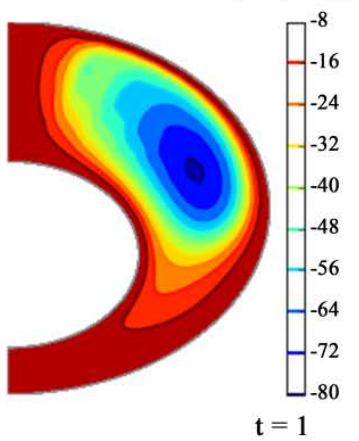

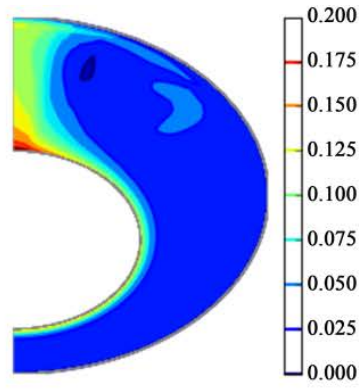

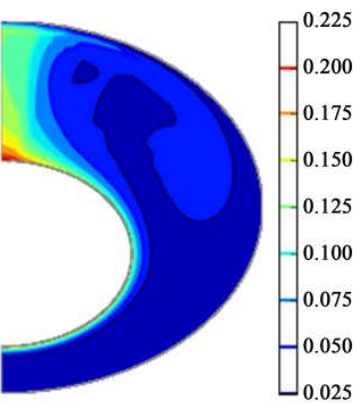

Figure 2. (a) Evolution of stramlines and isotherms for $e=-0.5$; (b) Evolution of stramlines and isotherms for $e=0$; (c) Evolution of stramlines and isotherms for $e=0.5$.

the internal wall and then goes down again along the external wall. When the modified Rayleigh number is high, isotherms are strongly deformed due to the motion. The maximum value of the stream function $\Psi_{\max }$ increase with the eccentricity. This increasing is more important with negative value of eccentricity. We noted the fluid vortex center upwards when eccentricity increases.

\subsection{Influence of Rayleigh Number}

Figures 3(a)-(c) show respectively the evolution of the Nusselt number, the stream function and the temperature of the heated wall.

Figure 3(a) displays the variation of average Nusselt number on the inner hemisphere against dimensionless time for differents Rayleigh numbers. We note a monotonous decrease of the average Nusselt number. The value of Rayleigh number at $10^{3}$ is more important than that obtained in the case of two spheres [8]. Starting from the expressions of the temperature and the modified Rayleigh, we can deduce the expression for the dimensionless temperature of the heated wall:

$$
\begin{gathered}
T=\frac{\lambda\left(T^{\prime}-T_{0}^{\prime}\right)}{q D} \\
q=\frac{v^{2} \lambda P r}{q \beta D^{4}} R a
\end{gathered}
$$

The dimensionless temperature is inversely proportional to the heat flux density applied on the internal hemisphere and thus to the Rayleigh number. Indeed, when the heat flux density increases, the convection also increases. We notice the 


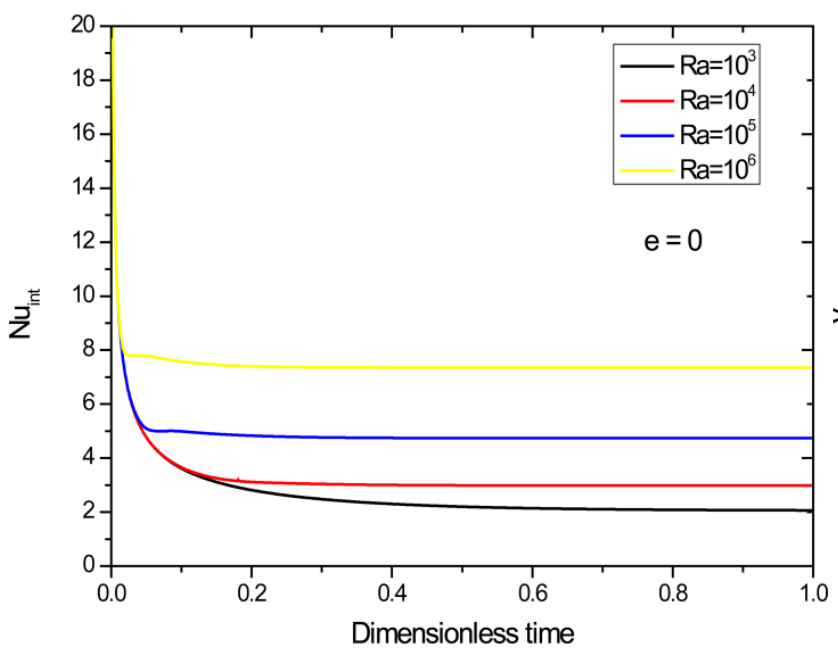

(a)

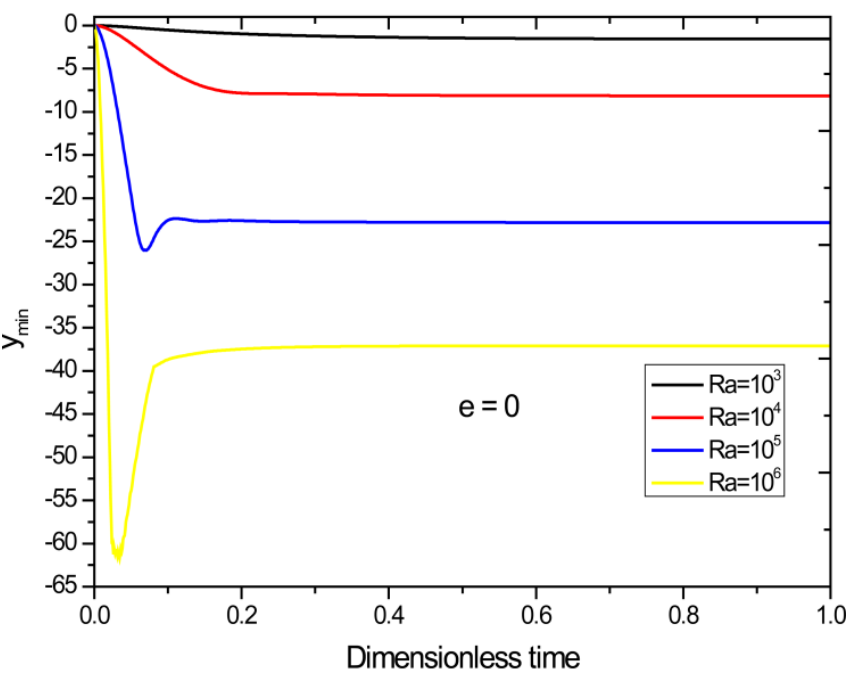

(b)

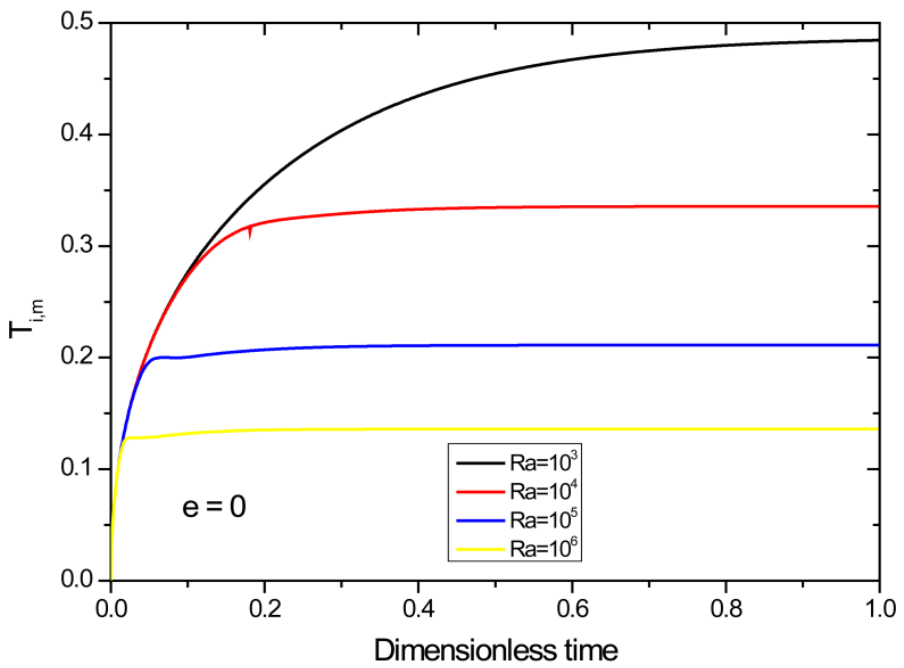

(c)

Figure 3. (a) Variation of average Nusselt number of the internal hemisphere according to the dimensionless time and various values of $R a$; (b) Variation of the minimum of stream function according to the dimensionless time and various values of $R a$; (c) Variation of the dimensionless mean temperature of the internal hemisphere with dimensionless time and various values of $R a$.

value of the dimensionless temperature is greater than that obtained with Sow [8].

Figure 3(c) shows also the variation of dimensionless mean temperature on the heated wall for various values of Rayleigh, which decrease as the Rayleigh number increases.

The time evolution of the minimum of stream function presented in Figure 3(b) for some Rayleigh values shows that the minimum of stream function decrease quickly over time and then it increases before stabilizing when the steady state is reached.

This is explaining by the fact at the beginning, the convection is predominant and the motion of fluid is important and the phenomena convection subside when the steady state is reached. 


\subsection{Influence of Eccentricity}

Figures 4(a)-(c) show respectively the evolution of the average Nusselt number, the minimum of stream function and the dimensionless temperature of the heated wall

Figure 4(a) displays, for three configurations, the time variation of the average Nusselt number one the internal hemisphere which decreases with eccentricity e when this one is negative and for the steady state. Besides, we note that for the same absolute values of eccentricty, the average Nusselt number is higher for positive values one.

Figure 4(b) correspond to influence of eccentricity on minimum stream function againt dimensionless time. That shows as the minimum of stream function decreases as the eccentricity increases.

Figure 4(c) shows the average temperature of the heated wall decreases with the eccentricity for negative values and increases with positive values thereof.

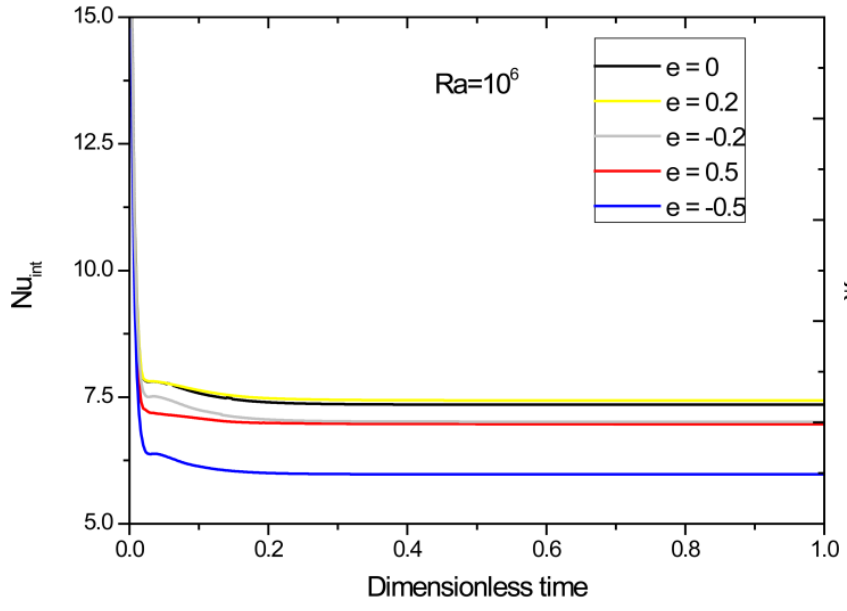

(a)

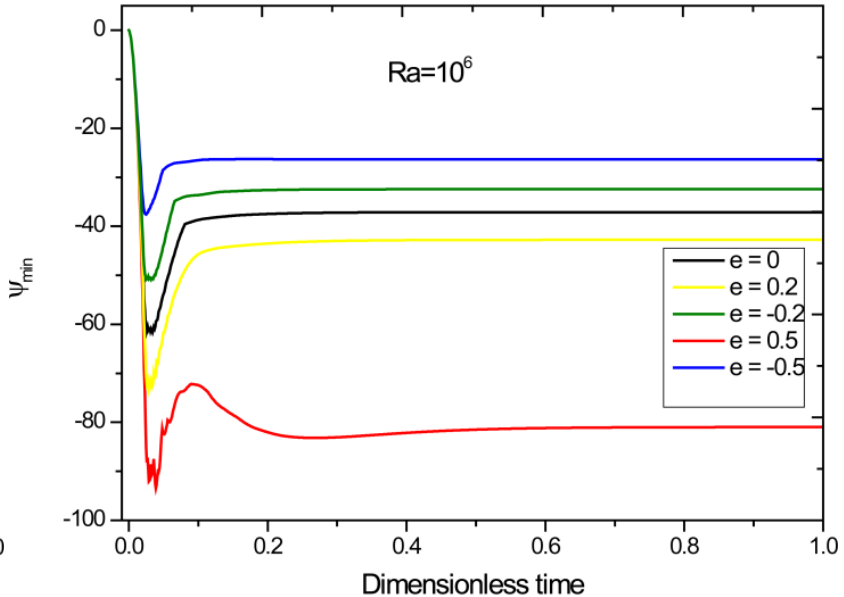

(b)

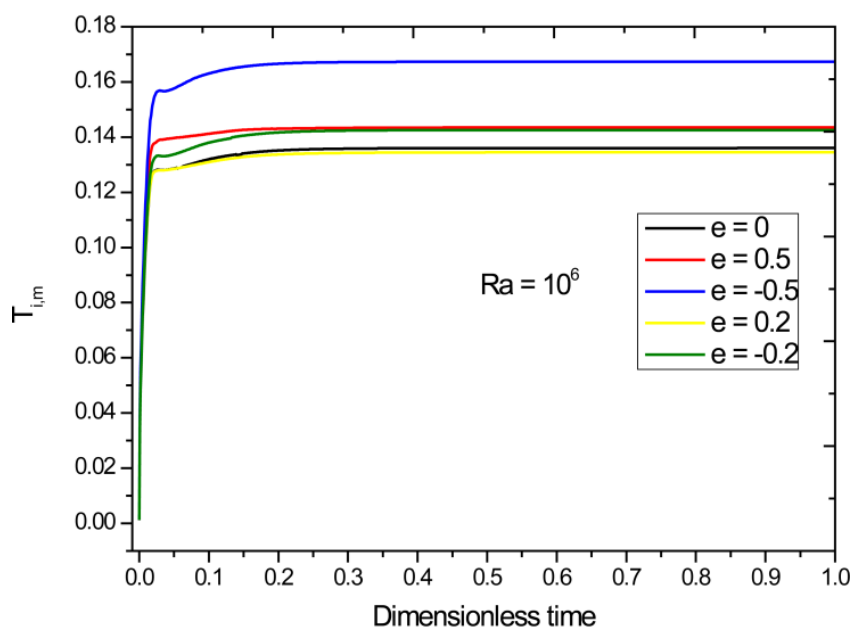

(c)

Figure 4. (a) Influence of eccentricity on the average Nusselt number of the heated wall according to the dimensionless time; (b) Influence of eccentricity on the minimum of stream function according to the dimensionless time; (c) Influence of eccentricity on the dimensionless of the average temperature according to the dimensionless time. 


\section{Conclusions}

The numerical study of unsteady natural convection between two eccentric hemispheres was investigated. The inner hemisphere is subjected to a heat flux constant while the outer one is maintained isotherm. The bispherical coordinates and the ADI and SOR methods based on finite difference method are used. The vorticity and stream-function variables are assumed.

The values obtained for the isotherms agree well with those of Sow [8]. We note a difference for those of the stream-function which may be due to the equation of stream-function which is not the same.

The study of the effects of eccentricity shows that the center of rotation moves toward the top of enclosure when the eccentricity increases. This increase is more important with negative value of eccentricity.

The average Nusselt number increases with the modified Rayleigh number. However, the dimensionless temperature decreases when the modified Rayleigh number increases regardless of the value of eccentricity and the minimum of stream function decreases as the eccentricity increases.

\section{Conflicts of Interest}

The authors declare no conflicts of interest regarding the publication of this paper.

\section{References}

[1] Chadwick, M.L., Webb, B.W. and Heaton, H.S. (1991) Natural Convection from Discrete Heat Sources in a Vertically Vented Rectangular Enclosure. Experimental Heat Transfer, 4, 199-216. https://doi.org/10.1080/08916159108946414

[2] Wilkes, J.O. and Churchill, S.W. (1966) The Finite-Difference Computation of Natural Convection in a Rectangular Enclosure. AIChE Journal, 12, 161-166. https://doi.org/10.1002/aic.690120129

[3] Orhan, A. and Yang, W.-J. (2000) Natural Convection in Enclosures with Localized Heating from Below and Symmetrical Cooling from Sides. International Journal of Numerical Methods for Heat \& Fluid Flow, 10, 518-529. https://doi.org/10.1108/09615530010338196

[4] Chen, W.R. (2005) Transient Natural Convection of Micropolar Fluids between Concentric and Vertically Eccentric Spheres. International Journal of Heat and Mass Transfer, 48, 1936-1951.

https://doi.org/10.1016/j.ijheatmasstransfer.2004.11.018

[5] Crawford, L. and Lemlich, R. (1962) Natural Convection in Horizontal Concentric Cylindrical Annuli. Industrial \& Engineering Chemistry Fundamentals, 1, 260-264. https://doi.org/10.1021/i160004a006

[6] Sarr, J., Mbow, C., Chehouani, H., Zeghmati, B., Benet, S. and Daguenet, M. (1995) Study of Natural Convection in an Enclosure Bounded by Two Concentric Cylinders and Two Diametric Planes. Journal of Heat Transfer, 117, 130-137. https://doi.org/10.1115/1.2822292

[7] Mack, L.R. and Hardee, H.C. (1968) Natural Convection between Concentric Spheres at Low Rayleigh Numbers. International Journal of Heat and Mass Transfer, 11. 387-396. https://doi.org/10.1016/0017-9310(68)90083-5 
[8] Mamadou, L.S. Joseph, S., Cheikh, M., Babacar, M., Bernard, C. and Mamadou, M.K. (2009) Geometrical and Rayleigh Number Effects in the Transient Laminar Free Convection between Two Vertically Eccentric Spheres. International Journal of Numerical Methods for Heat \& Fluid Flow, 19, 689-704. https://doi.org/10.1108/09615530910963599

[9] Lewandowski, W.M., Kubski, P.M., Khubeiz, J.M., Bieszk, H., Wilczewski, T. and Szymański, S. (1996) Theoretical and Experimental Study of Natural Convection Heat Transfer from Isothermal Hemisphere. International Journal of Heat and Mass Transfer, 40, 101-109. https://doi.org/10.1016/S0017-9310(96)00075-0

[10] Baïri, A., Monier-Vinard, E., Laraqi, N., Baïri, I., Nguyen, M.N. and Dia, C.T. (2014) Natural Convection in Inclined Hemispherical Cavities with Isothermal Disk and Dome Faced Downwards. Experimental and Numerical Study. Applied Thermal Engineering, 73, 1340-1347.

https://doi.org/10.1016/j.applthermaleng.2014.09.012

[11] Alilat, N. (2017) Natural Convective Heat Transfer in the Air-Filled Interstice between Inclined Concentric Hemispheres: Application to Thermoregulation in Electronics. International Journal of Numerical Methods for Heat \& Fluid Flow, 27, 2375-2384. https://doi.org/10.1108/HFF-10-2016-0392

[12] Moon, P. and Spencer, D.E. (1988) Field Theory Handbook: Including Coordinate Systems, Differential Equations and Their Solutions. 2nd Eidition, Berlin Heidelberg, Springer-Verlag.

[13] Peaceman, D.W. and Rachford Jr., H.H. (1955) The Numerical Solution of Parabolic and Elliptic Differential Equations. Journal of the Society for Industrial and Applied Mathematics, 3, 28-41. https://doi.org/10.1137/0103003

[14] Bejan, A. (2013) Convection Heat Transfer. John Wiley \& Sons, New York.

[15] Tazi-Charki, M.-N., Daoudi, S., Palec, G.L. and Daguenet, M. (1997) Étude numérique du modèle de Boussinesq de convection naturelle laminaire axisymétrique permanente dans un espace annulaire compris entre deux spheres. Revue Générale de Thermique, 36, 239-251. https://doi.org/10.1016/S0035-3159(97)80685-X 


\section{Nomenclature}

\section{Latin letters}

a, parameter of torus pole, $\mathrm{m}$

$e$, eccentricity $e=\frac{O_{i} O_{e}}{a}$

$g$, gravity intensity, $\mathrm{m} \cdot \mathrm{s}^{-2}$

$g_{1}$ and $g_{2}$ coefficients $g_{1}=g_{1}(\eta, \theta)=\frac{1-\cos \theta \operatorname{ch} \eta}{\operatorname{ch} \eta-\cos \theta}$

$g_{2}=g_{2}(\eta, \theta)=-\frac{\sin \theta \operatorname{sh} \eta}{\operatorname{ch} \eta-\cos \theta}$

$H$ and $K$, metrics coefficient dimensionless $H=\frac{1}{\operatorname{ch} \eta-\cos \theta}, \quad K=\frac{\sin \theta}{\operatorname{ch} \eta-\cos \theta}$

$N u_{e}$, Nusselt number for the outer hemisphere, $N u_{i}=\frac{1}{T_{i, m}}$

$N u_{e}$, Nusselt number for the inner hemisphere, $N u_{i}=\frac{1}{H_{e} T_{i, m}} \partial_{\eta} T$

$O_{i}$ and $O_{e}$, respectively center of inner and outer hemisphere

$\operatorname{Pr}$, Prandtl number $\operatorname{Pr}=\frac{v}{\alpha}$

$q$, Heat flux density $\mathrm{W} \cdot \mathrm{m}^{-2}$

$R_{i}$ and $R_{e}$, respectively radius of inner and outer hemisphere

$R a$, Rayleigh number, $R a=\frac{g \beta a^{4} q}{v \lambda \alpha}$

$t$, dimensionless time, $t=\frac{\alpha}{a^{2}} t^{\prime}$

$t^{\prime}$, dimension time, $s$

$T$, dimensionless temperature, $T=\frac{\lambda}{q a}\left(T^{\prime}-T_{0}^{\prime}\right)$

$U$ and $V$, dimensionless velocity components in the transformed planes $x$ and $y$, cartesian coordinates, $\mathrm{m}$

\section{Greek Symbols}

$\alpha$, thermal diffusivity, $\mathrm{m}^{2} \cdot \mathrm{s}^{-1}$

$\beta$, thermal expansion coefficient, $\mathrm{K}^{-1}$

$\Delta t$, time step, $\mathrm{s}$

$\Delta T$, difference of temperatures between the two hemispheres, $\mathrm{K}$

$\eta$ and $\theta$, bispherical coordinates $\mathrm{m}$

$\lambda$, thermal conductivity, $\mathrm{W} \cdot \mathrm{K}^{-1} \cdot \mathrm{m}^{-1}$

$v$, kinematical viscosity, $\mathrm{m}^{2} \cdot \mathrm{s}^{-1}$

$\Psi$, dimensionless stream-function, $\Psi=\frac{K}{\alpha a} \Psi^{\prime}$

$\Psi^{\prime}$, dimension stream-function

$\Omega$, dimensionless vorticity, $\Omega=\frac{a}{\alpha^{2}} \Omega^{\prime}$

$\Omega$, dimension vorticity 